\title{
Intrinsic state for an extended version of the interacting boson model
}

\author{
J. E. García-Ramos, ${ }^{1}$ J. M. Arias, ${ }^{1}$ J. Dukelsky, ${ }^{2}$ and P. Van Isacker ${ }^{3}$ \\ ${ }^{1}$ Departamento de Física Atómica, Molecular y Nuclear, Universidad de Sevilla, Apartado 1065, 41080 Sevilla, Spain \\ ${ }^{2}$ Instituto de Estructura de la Materia, Serrano 123, 28006 Madrid, Spain \\ ${ }^{3}$ Grand Accélérateur National d'Ions Lourds, Bô̂te Postale 5027, F-14076 Caen Cedex 5, France
}

(Received 16 July 1999; published 16 February 2000)

\begin{abstract}
An intrinsic-state formalism for the interacting boson model IBM-4 is presented. A basis of deformed bosons is introduced which allows the construction of a general trial wave function that has Wigner's spinisospin SU(4) symmetry as a particular limit. Intrinsic-state calculations are compared with exact ones, showing good agreement.
\end{abstract}

PACS number(s): 21.60.Fw, 21.30.Fe, 21.60.Ev

The interacting boson model (IBM) was originally proposed to describe collective low-lying states in even-even nuclei. The model building blocks are monopolar $(s)$ and quadrupolar $(d)$ bosons. In the original formulation of the model (IBM-1) no distinction was made between neutrons and protons [1]. Later, connections with the nuclear shell model were investigated $[2,3]$ and a new version was proposed in terms of neutron $\left(s_{\nu}, d_{\nu}\right)$ and proton $\left(s_{\pi}, d_{\pi}\right)$ bosons, known as IBM-2 [1]. The model has been widely applied to medium-mass and heavy nuclei, where neutrons and protons fill different major shells. In lighter nuclei with $N \approx Z$, however, neutrons and protons are in the same shell and a boson made of one neutron and one proton (known as a $\delta$ boson) should be included. This version of the boson model, called IBM-3 [4], is the simplest isospin invariant formulation of the IBM. The three types of bosons $(\nu, \pi$, and $\delta$ ) form an isospin $T=1$ triplet and correspond, microscopically, to spatially symmetric nucleon pairs with $S=0$. In particular, the $\delta$ boson corresponds to a spatially symmetric $S=0$ neutron-proton pair. A further extension of the IBM introduces the neutron-proton boson with $T=0$, or $\sigma$ boson, corresponding to a spatially symmetric nucleon pair with $S$ $=1$. This version is known as IBM-4 [5] and gives a proper description of even-even as well as odd-odd $N \approx Z$ nuclei.

The IBM-3 and IBM-4 are appropriate models for $N \approx Z$ nuclei approaching the proton drip line. Such nuclei are under intensive study at the moment, in particular with radioactive nuclear beams. In addition, the IBM-4 is a reasonably simple, yet detailed model to study the competition between $T=0$ and $T=1$ pairing, one of the hot topics in present-day nuclear structure physics.

All versions of the IBM are algebraic in nature and do not have a direct geometrical interpretation. Such interpretation can be achieved, however, by introducing an intrinsic state that provides a connection to geometric models such as that of Bohr and Mottelson [6]. Intrinsic states have been proposed for IBM-1 [7-10], IBM-2 [11-13], and IBM-3 $[14,15]$. Their primary use is to provide a geometric visualization of the model. In addition, a considerable reduction is achieved in the complexity of calculations, which leaves room for the inclusion of extra degrees of freedom.

The purpose of this paper is to propose an intrinsic state for IBM-4. In the limit of strong isovector pairing it reduces to the intrinsic state for IBM-3; in general, it can be used for studying the competition between $T=0$ and $T=1$ pairing in $N \approx Z$ nuclei. First, the mean-field formalism for IBM-4 is presented. This formalism is subsequently checked against the results of an exact calculation.

The ensemble of bosons in the IBM-4 consists of isovector $T=1$ and isoscalar $T=0$ bosons which have intrinsic spin $S=0$ and $S=1$, respectively, to ensure spatial symmetry. The allowed spin-isospin combinations are thus $(T, S)$ $=(1,0)$ and $(T, S)=(0,1)$. These, together with the orbital angular momenta $l=0,2$, give rise to 36 different bosons. The corresponding boson creation and annihilation operators are $\gamma_{l m, T \tau, S \sigma}^{\dagger}$ and $\gamma_{l m, T \tau, S \sigma}$ where $l$ is the orbital angular momentum, $m$ is its projection, $T$ is the isospin, $\tau$ is its projection, $S$ is the spin, and $\sigma$ is its projection. The operators $\tilde{\gamma}_{l m, T \tau, S \sigma}=(-1)^{l+T+S-m-\tau-\sigma} \gamma_{l-m, T-\tau, S-\sigma}$ are introduced because of their appropriate tensor transformation properties.

The construction of an intrinsic state requires two ingredients. First, it needs a basis of deformed bosons and secondly, it requires a trial wave function. The deformed bosons are defined in terms of the spherical ones through a unitary Hartree-Bose transformation,

$$
\begin{gathered}
\Omega_{p, T \tau, S \sigma}^{\dagger}=\sum_{l m} \lambda_{l m}^{p T \tau S \sigma} \gamma_{l m, T \tau, S \sigma}^{\dagger}, \\
\gamma_{l m, T \tau, S \sigma}^{\dagger}=\sum_{p} \lambda_{l m}^{p T \tau S \sigma *} \Omega_{p, T \tau, S \sigma}^{\dagger},
\end{gathered}
$$

and their Hermitian conjugates. The deformation parameters $\lambda$ in these equations satisfy the following orthonormalization relations:

$$
\begin{gathered}
\sum_{l m} \lambda_{l m}^{p^{\prime} T \tau S \sigma *} \lambda_{l m}^{p T \tau S \sigma}=\delta_{p p^{\prime}}, \\
\sum_{p} \lambda_{l m}^{p T \tau S \sigma *} \lambda_{l^{\prime} m^{\prime}}^{p T \tau S \sigma}=\delta_{l l^{\prime}} \delta_{m m^{\prime}} .
\end{gathered}
$$

For convenience, a global label $\xi$ is used for spin, isospin, and their projections, $\xi \equiv(T \tau S \sigma)$. This new index plays the same role in IBM-4 as the isospin projection $\tau$ does in the IBM-3 intrinsic-state formalism [15]. The index $p$ labels the different deformed bosons. The fundamental deformed boson 
has $p=0$ while excited ones have $p=1,2, \ldots, \Sigma_{l}(2 l+1)$ -1 . If only $s$ and $d$ bosons are included, the maximum value of $p$ is 5. Only the ground-state properties are considered here; so the superscript $p$ is always zero and can be omitted henceforth.

The definition of the ground-state trial wave function follows Ref. [15]; it is different depending on whether the system is even-even or odd-odd. For even-even nuclei with proton excess (the case of neutron excess is obtained by interchanging $N_{p}$ and $N_{n}$ ) the proposed trial wave function for the ground state has the form

$$
\left|\phi\left(\delta, \alpha_{T}, \alpha_{S}\right)\right\rangle_{e e}=\Delta^{\dagger N_{n}}\left(\delta, \alpha_{T}, \alpha_{S}\right) \Omega_{T=1 \tau=1}^{\dagger N_{p}-N_{n}}|0\rangle,
$$

where $N_{n}\left(N_{p}\right)$ is half the number of valence neutrons (protons) and

$$
\begin{aligned}
\Delta^{\dagger}\left(\delta, \alpha_{T}, \alpha_{S}\right)= & \left(\Omega_{T=1 \tau=1}^{\dagger} \Omega_{T=1 \tau=-1}^{\dagger}\right. \\
& \left.+\alpha_{T} \Omega_{T=1 \tau=0}^{\dagger} \Omega_{T=1 \tau=0}^{\dagger}\right) \\
& +\delta\left(\Omega_{S=1 \sigma=1}^{\dagger} \Omega_{S=1 \sigma=-1}^{\dagger}\right. \\
& \left.+\alpha_{S} \Omega_{S=1 \sigma=0}^{\dagger} \Omega_{S=1 \sigma=0}^{\dagger}\right) .
\end{aligned}
$$

The description of odd-odd nuclei is complicated even in the ground state, since in general their spin-isospin values are not known a priori. In $N=Z$ nuclei, which is the case of most interest, those values are known, being either $(T, S)$ $=(1,0)$ or $(T, S)=(0,1)$. Correspondingly, two trial wave functions are proposed:

$\left|\phi\left(\delta, \alpha_{T}, \alpha_{S}\right)\right\rangle_{o o-1}=\Delta^{\dagger N_{n}-1 / 2}\left(\delta, \alpha_{T}, \alpha_{S}\right) \Omega_{T=1 \tau=1}^{\dagger}|0\rangle$

and

$\left|\phi\left(\delta, \alpha_{T}, \alpha_{S}\right)\right\rangle_{o o-2, \sigma}=\Delta^{\dagger N_{n}-1 / 2}\left(\delta, \alpha_{T}, \alpha_{S}\right) \Omega_{S=1 \sigma}^{\dagger}|0\rangle$.

Which of these two states is lower in energy depends on a delicate balance of the different terms in the Hamiltonian which in turn follow from the competition between $T=0$ and $T=1$ pairing.

In addition to the deformation parameters, three variational parameters, $\alpha_{T}, \alpha_{S}$, and $\delta$, appear in the trial wave functions. The first two are related to isospin and spin symmetry breaking in the trial wave function. For deformation parameters independent of $\xi$ and for $\alpha_{T}=\alpha_{S}=-1 / 2$, the operator $\Delta^{\dagger}\left(\delta, \alpha_{T}, \alpha_{S}\right)$ corresponds to a bosonic pair scalar in spin and isospin. The parameter $\delta$ measures the relative importance of isovector and isoscalar bosons in the ground state. In the limit of $\delta=0$, the number of isoscalar bosons in the ground state is zero and the IBM-3 trial state is recovered [15]. Another interesting limit is $\delta= \pm 1$ which is obtained if the IBM-4 Hamiltonian has Wigner's SU(4) symmetry [5]. In this case $T=0$ and $T=1$ bosons are treated on an equal footing.

Given a general IBM-4 Hamiltonian $\hat{H}$, the ground-state equilibrium parameters are obtained by minimizing the expectation value of the Hamiltonian in the appropriate trial wave function (3), (5), and (6). A general expression for this expectation value can be written as

$$
\begin{aligned}
& E\left(\lambda, \delta, \alpha_{T}, \alpha_{S}\right) \\
& =\sum_{\xi_{1} \xi_{2}} \epsilon_{\xi_{1} \xi_{2}} \widetilde{f}_{1}\left(\delta, \alpha_{T}, \alpha_{S}, \xi_{1} \xi_{2}\right) \\
& \quad+\sum_{\xi_{1} \xi_{2} \xi_{3} \xi_{4}} V_{\xi_{1}, \xi_{2}, \xi_{3}, \xi_{4}}^{c} \widetilde{f}_{2}\left(\delta, \alpha_{T}, \alpha_{S}, \xi_{1} \xi_{2} \xi_{3} \xi_{4}\right),
\end{aligned}
$$

where

$$
\boldsymbol{\epsilon}_{\xi_{1}, \xi_{2}}=\sum_{l_{1} m_{1} l_{2} m_{2}} \tilde{\varepsilon}_{l_{1} m_{1} \xi_{1} l_{2} m_{2} \xi_{2}} \lambda_{l_{1} m_{1}}^{\xi_{2}^{*}} \lambda \lambda_{l_{2} m_{2}}^{\xi_{2}}
$$

$$
\begin{aligned}
& V_{\xi_{1}, \xi_{2}, \xi_{3}, \xi_{4}}^{c} \\
& =\sum_{l_{1} m_{1} l_{2} m_{2} l_{3} m_{3} l_{4} m_{4}} V_{l_{1} m_{1} \xi_{1}, l_{2} m_{2} \xi_{2}, l_{3} m_{3} \xi_{3}, l_{4} m_{4} \xi_{4}} \\
& \times \lambda_{l_{1} m_{1}}^{\xi_{1}^{*}} \lambda_{l_{2} m_{2}}^{\xi_{2} *} \lambda_{l_{3} m_{3}}^{\xi_{3}} \lambda \lambda_{l_{4} m_{4}}^{\xi_{4}},
\end{aligned}
$$

$$
\begin{aligned}
& \tilde{f}_{1}\left(\delta, \alpha_{T}, \alpha_{S}, \xi_{1} \xi_{2}\right) \\
&=\delta_{\xi_{1} \xi_{2}} \frac{\left\langle\phi\left(\delta, \alpha_{T}, \alpha_{S}\right)\left|\Omega_{\xi_{1}}^{\dagger} \Omega_{\xi_{2}}\right| \phi\left(\delta, \alpha_{T}, \alpha_{S}\right)\right\rangle}{\left\langle\phi\left(\delta, \alpha_{T}, \alpha_{S}\right) \mid \phi\left(\delta, \alpha_{T}, \alpha_{S}\right)\right\rangle}
\end{aligned}
$$

and

$$
\begin{aligned}
\tilde{f}_{2}\left(\delta, \alpha_{T}, \alpha_{S}, \xi_{1} \xi_{2} \xi_{3} \xi_{4}\right) & \\
= & \frac{\left\langle\phi\left(\delta, \alpha_{T}, \alpha_{S}\right)\left|\Omega_{\xi_{1}}^{\dagger} \Omega_{\xi_{2}}^{\dagger} \Omega_{\xi_{3}} \Omega_{\xi_{4}}\right| \phi\left(\delta, \alpha_{T}, \alpha_{S}\right)\right\rangle}{\left\langle\phi\left(\delta, \alpha_{T}, \alpha_{S}\right) \mid \phi\left(\delta, \alpha_{T}, \alpha_{S}\right)\right\rangle} .
\end{aligned}
$$

The isospin matrix elements (10) and (11) are calculated straightforwardly from a binomial expansion of the trial wave function. Furthermore, the parameters $\tilde{\varepsilon}_{l_{1} m_{1} \xi_{1} l_{2} m_{2} \xi_{2}}$ and $V_{l_{1} m_{1} \xi_{1}, l_{2} m_{2} \xi_{2}, l_{3} m_{3} \xi_{3}, l_{4} m_{4} \xi_{4}}$ in Eqs. (8) and (9) are defined as

$$
\tilde{\varepsilon}_{l_{1} m_{1} \xi_{1} l_{2} m_{2} \xi_{2}} \equiv\left\langle l_{1} m_{1} \xi_{1}|\hat{H}| l_{2} m_{2} \xi_{2}\right\rangle
$$

$$
\begin{aligned}
V_{l_{1} m_{1} \xi_{1}, l_{2} m_{2} \xi_{2}, l_{3} m_{3} \xi_{3}, l_{4} m_{4} \xi_{4}} \\
\equiv \\
\quad \frac{1}{4}\left\langle l_{1} m_{1} \xi_{1}, l_{2} m_{2} \xi_{2}|\hat{V}| l_{3} m_{3} \xi_{3}, l_{4} m_{4} \xi_{4}\right\rangle \\
\quad \times \sqrt{1+\delta_{l_{1} l_{2}} \delta_{m_{1} m_{2}} \delta_{\xi_{1} \xi_{2}}} \sqrt{1+\delta_{l_{3} l_{4}} \delta_{m_{3} m_{4}} \delta_{\xi_{3} \xi_{4}}},
\end{aligned}
$$

where $\hat{V}$ stands for the two-body terms in the Hamiltonian $\hat{H}$. It is worth noting that $\widetilde{\varepsilon}$ is not diagonal in $l$.

The energy (7) depends explicitly on the deformation parameters $\lambda$ and implicitly on $\alpha_{T}, \alpha_{S}$, and $\delta$ through $\widetilde{f}_{1}$ and 
$\tilde{f}_{2}$. The deformation parameters $\lambda$ are obtained by minimizing the energy with the constraint of conserving the transformation norm,

$$
\delta\left[E\left(\lambda, \delta, \alpha_{T}, \alpha_{S}\right)-\sum_{\xi l m} E_{\xi} \lambda_{l m}^{\xi *} \lambda_{l m}^{\xi}\right]=0 .
$$

The Hartree-Bose equations for IBM-4 are obtained by deriving with respect to $\lambda_{l m}^{\xi *}$,

$$
\sum_{l_{2} m_{2}} h_{l_{1} m_{1}, l_{2} m_{2}}^{\xi} \lambda_{l_{2} m_{2}}^{\xi}=E_{\xi} \lambda_{l_{1} m_{1}}^{\xi}
$$

where the Hartree-Bose matrix $h^{\xi}$ is

$$
\begin{aligned}
h_{l_{1} m_{1}, l_{2} m_{2}}^{\xi}= & \tilde{\varepsilon}_{l_{1} m_{1} \xi l_{2} m_{2} \xi} \tilde{f}_{1}\left(\delta, \alpha_{T}, \alpha_{S}, \xi \xi\right) \delta_{m_{1} m_{2}} \\
& +2 \sum_{l_{3} m_{3} l_{4} m_{4} \xi_{2} \xi_{3} \xi_{4}} V_{l_{1} m_{1} \xi, l_{3} m_{3} \xi_{3}, l_{4} m_{4} \xi_{4}, l_{2} m_{2} \xi_{2}} \\
& \times \frac{\lambda_{l_{3} m_{3}}^{\xi_{3}^{*}} \lambda_{l_{4} m_{4}}^{\xi_{4}} \lambda_{l_{2} m_{2}}^{\xi_{2}}}{\lambda_{l_{2} m_{2}}^{\xi}} \tilde{f}_{2}\left(\delta, \alpha_{T}, \alpha_{S}, \xi \xi_{3} \xi_{4} \xi_{2}\right) .
\end{aligned}
$$

There are six coupled equations of this form, which are solved for fixed values of $\alpha_{T}, \alpha_{S}$, and $\delta$ in a self-consistent way. This procedure yields the equilibrium deformation parameters $\lambda$. The equilibrium values for the parameters $\alpha_{T}$, $\alpha_{S}$, and $\delta$ are obtained by an additional minimization. In fact, if the deformation parameters are independent of $\xi$, the parameters $\alpha_{T}$ and $\alpha_{S}$ can be fixed to the value $-1 / 2$, which corresponds, as mentioned above, to a state with welldefined spin and isospin. As was shown for IBM-3 [14,15], this is a good approximation for $N=Z$ nuclei.

To test the present formalism, comparisons with exact calculations are carried out. Numerical calculations in the framework of IBM-4 are now possible [16] but still difficult. Also, only few dynamical limits have been studied. Here the following schematic Hamiltonian is considered:

$\hat{H}=a \hat{C}_{2}\left[\mathrm{SU}_{T S}(4)\right]+b \hat{C}_{2}\left[\mathrm{SU}_{S}(3)\right]+c \hat{C}_{2}\left[\mathrm{SU}_{L}(3)\right]$,

where $\hat{C}_{2}[G]$ stands for the quadratic Casimir operator of the algebra $G$. The first operator is an invariant of the algebra $\mathrm{SU}_{T S}(4)$ which is the boson equivalent of Wigner's supermultiplet algebra [5]. It is worth noting here that, as mentioned in [18], there are two alternative $\mathrm{SU}_{T S}(4)$ limits with the same eigenspectrum but different phases in the wave function. The results presented below are obtained within one of them, which is associated with the election of the operator $\hat{Y}_{\mu \nu}^{+}$(using the notation of Ref. [18]). The use of the alternative limit, using $\hat{Y}_{\mu \nu}^{-}$, gives identical results but with a different sign for $\delta$. The second operator in Eq. (17) is an invariant of the $\mathrm{SU}_{S}(3)$ algebra associated with the $\sigma(S$ $=1, T=0$ ) boson. (Its definition is analogous to that of $\mathrm{SU}_{S}(3)$ considered in the $L=0$ IBM-4 of Ref. [18].) The last
TABLE I. Exact and mean-field energies of ground states, and their isospins, for selected values of $b / a$. The cases shown correspond to $N=Z$ odd-odd nuclei with $N=5$ and $N=15$ bosons, respectively.

\begin{tabular}{ccccccc}
\hline \hline & \multicolumn{2}{c}{$E_{\mathrm{gs}} / a(N=5)$} & & \multicolumn{2}{c}{$E_{\mathrm{gs}} / a(N=15)$} \\
\cline { 3 - 5 }$b / a$ & $T$ & Exact & Mean field & Exact & Mean field \\
\hline-1.0 & 0 & -27.8359 & -27.8357 & -236.126 & -236.125 \\
-0.8 & 0 & -20.5261 & -20.5257 & -183.938 & -183.937 \\
-0.6 & 0 & -13.4392 & -13.4383 & -132.395 & -132.394 \\
-0.4 & 0 & -6.69081 & -6.68917 & -81.9874 & -81.9861 \\
-0.2 & 0 & -0.46290 & -0.46140 & -34.0488 & -34.0484 \\
0.0 & 0,1 & 5.00000 & 5.00000 & 5.00000 & 5.00000 \\
0.2 & 1 & 6.56989 & 6.57374 & 11.8499 & 11.9770 \\
0.4 & 1 & 7.76803 & 7.78117 & 15.2339 & 15.4644 \\
0.6 & 1 & 8.70462 & 8.72895 & 17.5636 & 17.8459 \\
0.8 & 1 & 9.45690 & 9.49143 & 19.3676 & 19.6743 \\
1.0 & 1 & 10.0763 & 10.1187 & 20.8479 & 21.1652 \\
\hline \hline
\end{tabular}

invariant in Eq. (17) is an orbital deformation term associated with an $\mathrm{SU}(3)$ algebra which is scalar in spin and isospin.

With this Hamiltonian three situations are studied. The first case corresponds to $a \neq 0$ and $c=0$. In Ref. [17] the competition of $T=0$ and $T=1$ pairing was discussed using this Hamiltonian. This is a relevant test for the formalism presented here since it explores the spin-isospin degrees of freedom, which represent the main difference of IBM-4 with respect to previous versions of the IBM. In this case the mean-field and exact calculations are almost identical although the exact calculation is always slightly lower in energy. This can be appreciated in Table I where exact and mean-field ground-state energies (in units of $a$ ) are given for $N=5$ and $N=15$ bosons for selected values of $b / a$. The value $b=0$ yields a Hamiltonian with the SU(4) symmetry, and degenerate lowest $T=0$ and $T=1$ states. Negative values of the ratio $b / a$ favor $T=0$ while positive values favor $T=1$ pairing. The expectation value of the schematic Hamiltonian (17) with $c=0$ is independent of the deformation parameters. The minimum of the energy occurs for $\xi$ independent parameters and $\alpha_{T}=\alpha_{S}=-\frac{1}{2}$. This is so because the energy has no orbital dependence. It is worth noting that the variational wave functions (3)-(6) contain for special values of $\delta$ the lowest eigenfunctions of the $\mathrm{SU}_{T}(3) \otimes \mathrm{SU}_{S}(3)$ limit [18] $(\delta=\infty)$ and of the $\operatorname{SU}_{T S}(4)$ limit [18] $(\delta=-1)$.

The second case corresponds to $a \neq 0$ and $b=0$ and includes the deformation term $\hat{C}_{2}\left[\mathrm{SU}_{L}(3)\right]$. The exact groundstate energy is known analytically:

$$
E=a \lambda(\lambda+4)+c 2 N(2 N+3) .
$$

This is so because, for sufficiently large negative $c$, the ground state belongs to the $\mathrm{SU}_{T S}(4)$ representation $(0, \lambda, 0)$ and the $\mathrm{SU}_{L}(3)$ representation $(2 N, 0)$, where $N$ is the boson number and $\lambda=T$ for even-even nuclei and $\lambda=1$ for oddodd $N=Z$ nuclei [18]. The corresponding calculation is also 
performed with the mean-field formalism presented here and the exact results are reproduced. The calculation gives an intrinsic state with $\beta_{\xi} \equiv \lambda_{20}^{\xi} / \lambda_{00}^{\xi}=\sqrt{2}, \alpha_{T}=\alpha_{S}=-\frac{1}{2}$, and $\delta$ $=-1$, which is an eigenstate of the Hamiltonian (17) with $a \neq 0$ and $b=0$. This result is similar to that obtained for the intrinsic state of IBM-1 [19].

The last case considered is the general one with $a, b$, and $c$ different from zero. The ground state still belongs to the $\mathrm{SU}_{L}(3)$ representation $(2 N, 0)$ and hence the contribution to the ground-state energy coming from $c \hat{C}_{2}\left[\mathrm{SU}_{L}(3)\right]$ is diagonal. The other two terms in the Hamiltonian can be diagonalized as in Ref. [17]. Thus the exact energies are those calculated in Table I (under "Exact'") plus $c 2 N(2 N+3)$. This calculation is repeated with the mean-field formalism and produces an intrinsic state with the same $\mathrm{SU}_{L}(3)$ symmetry as the exact one, $(2 N, 0)$. As in the preceding case $\beta_{\xi} \equiv \lambda_{20}^{\xi} / \lambda_{00}^{\xi}=\sqrt{2}, \quad \alpha_{T}=\alpha_{S}=-\frac{1}{2}$, but now $\delta \neq-1$. The mean-field energies are those given in Table I (under "Mean field') plus $c 2 N(2 N+3)$.

These results demonstrate that the present mean-field formalism has the correct ingredients for reproducing the full IBM-4 calculation. In addition, this formalism allows calcu- lations for an arbitrary number of bosons and a general Hamiltonian, not necessarily corresponding to a dynamical symmetry limit of the model.

To summarize, a Hartree-Bose mean-field approximation for IBM-4 has been presented, along with trial wave functions valid for even-even and odd-odd nuclei with $N=Z$. The trial wave functions include boson correlations in the spin and isospin spaces. Comparisons with exact calculations show good agreement from which it can be inferred that the present formalism gives a good approximation to the full diagonalization. The aim is now to consider more realistic IBM-4 Hamiltonians that include both types of pairing ( $T$ $=0$ and $T=1$ ), and a spin-orbit coupling as well as more general quadrupole deformation terms. This will enable the study of the interplay between single-particle, spin-isospin, and orbital degrees of freedom. This work is currently in progress.

We wish to thank S. Pittel and D. D. Warner for useful comments. This work has been supported by the Spanish DGICYT under Contract Nos. PB98-1111 and PB95-0123 and a DGICYT-IN2P3 agreement.
[1] F. Iachello and A. Arima, The Interacting Boson Model (Cambridge University Press, Cambridge, 1987).

[2] T. Otsuka, A. Arima, F. Iachello, and I. Talmi, Phys. Lett. 76B, 139 (1978).

[3] F. Iachello and I. Talmi, Rev. Mod. Phys. 59, 339 (1987).

[4] J. P. Elliott and A. P. White, Phys. Lett. 97B, 169 (1980).

[5] J. P. Elliott and J. A. Evans, Phys. Lett. 101B, 216 (1981).

[6] A. Bohr and B.R. Mottelson, Nuclear Structure II. Nuclear Deformations (Benjamin, New York 1975).

[7] J. N. Ginocchio and M. W. Kirson, Nucl. Phys. A350, 31 (1980)

[8] J. N. Ginocchio and M. W. Kirson, Phys. Rev. Lett. 44, 1744 (1980).

[9] A. E. L. Dieperink and O. Scholten, Nucl. Phys. A346, 125 (1980).

[10] J. Dukelsky, G. G. Dussel, R. P. J. Perazzo, S. L. Reich, and H.
M. Sofia, Nucl. Phys. A425, 93 (1984).

[11] A. Leviatan and M. W. Kirson, Ann. Phys. (N.Y.) 201, 13 (1990).

[12] J. N. Ginocchio and A. Leviatan, Ann. Phys. (N.Y.) 216, 152 (1992).

[13] S. Pittel and J. Dukelsky, Phys. Rev. C 32, 335 (1985).

[14] J. N. Ginocchio and A. Leviatan, Phys. Rev. Lett. 73, 1903 (1994).

[15] J. E. García-Ramos, J. M. Arias, J. Dukelsky, E. Moya de Guerra, and P. Van Isacker, Phys. Rev. C 57, R479 (1998).

[16] O. Juillet (unpublished).

[17] P. Van Isacker and D. D. Warner, Phys. Rev. Lett. 78, 3266 (1997).

[18] P. Van Isacker, J. Dukelsky, S. Pittel, and O. Juillet, J. Phys. G 24, 1261 (1998).

[19] A. Leviatan, Phys. Rev. Lett. 77, 818 (1996). 